\title{
Clinical, pathological, and radiological characteristics of solitary ground-glass opacity lung nodules on high-resolution computed tomography
}

This article was published in the following Dove Press journal:

Therapeutics and Clinical Risk Management

20 September 2016

Number of times this article has been viewed

\section{Zhi-Xin Qiu' \\ Yue Cheng' \\ Dan Liu' \\ Wei-Ya Wang ${ }^{2}$ \\ $\mathrm{Xia} \mathrm{Wu}^{2}$ \\ Wei-Lu Wu² \\ Wei-Min $\mathrm{Li}^{1,2}$}

'Department of Respiratory Medicine, ${ }^{2}$ Department of Pathology, West China Hospital, Sichuan University, Chengdu, People's Republic of China
Correspondence: Wei-Min Li Department of Respiratory Medicine, West China Hospital, Sichuan University, Chengdu 61004I, People's Republic of China Tel +862885423998

Email weimi003@yahoo.com
Background: Lung nodules are being detected at an increasing rate year by year with highresolution computed tomography (HRCT) being widely used. Ground-glass opacity nodule is one of the special types of pulmonary nodules that is confirmed to be closely associated with early stage of lung cancer. Very little is known about solitary ground-glass opacity nodules (SGGNs). In this study, we analyzed the clinical, pathological, and radiological characteristics of SGGNs on HRCT.

Methods: A total of 95 resected SGGNs were evaluated with HRCT scan. The clinical, pathological, and radiological characteristics of these cases were analyzed.

Results: Eighty-one adenocarcinoma and 14 benign nodules were observed. The nodules included 12 (15\%) adenocarcinoma in situ (AIS), 14 (17\%) minimally invasive adenocarcinoma (MIA), and 55 (68\%) invasive adenocarcinoma (IA). No patients with recurrence till date have been identified. The positive expression rates of anaplastic lymphoma kinase and ROS-1 (proto-oncogene tyrosine-protein kinase ROS) were only $2.5 \%$ and $8.6 \%$, respectively. The specificity and accuracy of HRCT of invasive lung adenocarcinoma were $85.2 \%$ and $87.4 \%$. The standard uptake values of only two patients determined by $18 \mathrm{~F}-\mathrm{FDG}$ positron emission tomography/computed tomography (PET/CT) were above 2.5. The size, density, shape, and pleural tag of nodules were significant factors that differentiated IA from AIS and MIA. Moreover, the size, shape, margin, pleural tag, vascular cluster, bubble-like sign, and air bronchogram of nodules were significant determinants for mixed ground-glass opacity nodules (all $P<0.05)$.

Conclusion: We analyzed the clinical, pathological, and radiological characteristics of SGGNs on HRCT and found that the size, density, shape, and pleural tag of SGGNs on HRCT are found to be the determinant factors of IA. In conclusion, detection of anaplastic lymphoma kinase expression and performance of PET/CT scan are not routinely recommended.

Keywords: SGGNs, HRCT, adenocarcinoma, clinical, pathological

\section{Introduction}

With the increased use of high-resolution computed tomography (HRCT) for screening lung diseases, very faint and smaller lesions known as ground-glass opacity nodules (GGNs) are being encountered more frequently. GGNs are characterized as lesions of homogenous density and with hazy increase in density in the lung field that does not obscure the bronchovascular structure. ${ }^{1-3}$ They were classified into pure GGNs (pGGNs) and mixed GGNs (mGGNs), and the frequency of malignancy was higher for mGGNs than for pGGNs among patients who underwent low-dose CT scan. ${ }^{4}$ 
Although some studies have reported the characteristics of pGGNs and their history, ${ }^{5,6}$ there is no report about solitary GGNs (SGGNs) on HRCT scan. Therefore, the purpose of our research was to compare the clinical pathology with morphologic features of patients with SGGNs $\leq 30 \mathrm{~mm}$ in diameter on HRCT.

\section{Materials and methods Subjects}

We retrospectively reviewed all HRCT scan reports for SGGNs that were taken from January 2008 to March 2015 in the radiology department of West China Hospital and identified patients who underwent pathological biopsy after the surgery. One thoracic radiologist reviewed all the HRCT images again to identify the selected patients with persistent SGGNs before surgically removed, and collected all the preoperative HRCT images at the same time. Our inclusion criteria were as follows: 1) lesions with a thickness of $\leq 1 \mathrm{~mm}$ on HRCT scan, 2) SGGNs with a maximum diameter of $\leq 30 \mathrm{~mm}$, and 3) patients who had their lesions surgically removed and had pathological diagnosis. Ethical approval was obtained from the Ethics Committee of West China Hospital, patients' confidentiality was maintained. The Ethics Committee of West China Hospital deemed patient consent not necessary.

\section{Evaluation of nodule morphology using HRCT scan}

CT scans were obtained with SIEMENS SOMATOM ${ }^{\circledR}$ Definition Flash scanners (Munich, Germany). The following parameters were used to obtain HRCT images: collimator with $64 \times 0.6 \mathrm{~mm}$, section thickness of $1 \mathrm{~mm}$, reorganization interval of $0.66 \mathrm{~mm}$, and tube voltage of $120 \mathrm{kV}$. Tube current is calculated according to an individual's weight, height, and body mass index. The tube current was $220 \mathrm{mAs}$ for body mass index $\leq 25 \mathrm{~kg} / \mathrm{m}^{2}$ and $330 \mathrm{mAs}$ for body mass index $>25 \mathrm{~kg} / \mathrm{m}^{2}$. Two chest radiologists, each with more than 2 years of experience in diagnosing thoracic disease, assessed nodule morphology blindly and independently. Morphology included density, size (the maximum diameter), shape (round, oval, or irregular), margin (smooth, lobular, spiculated, or lobular and spiculated), pleural tag, vascular cluster, bubble lucency, and air bronchogram. The average values of size were obtained by two observers independently.

\section{Surgery and histopathologic evaluation}

All specimens were designated $\mathrm{R} 0$ (no residual tumor at the primary tumor site after surgical resection). Adenocarcinomas were histologically classified according to the criteria of Lung Cancer/American Thoracic Society/European Respiratory Society International Multidisciplinary Lung Adenocarcinoma Classification ${ }^{7}$ by two lung pathologists (Wei-Ya Wang and Wei-Lu Wu, each with more than 5 years of experience in lung pathology).

\section{Treatment and follow-up evaluation}

A total of 81 adenocarcinoma patients were included for analysis. All the SGGNs were removed by lobectomy $(n=46)$, sublobar resection $(n=21)$, and wedge resection $(n=14)$. Every patient was followed up for chest X-ray post-surgery, and some were regularly followed up for chest CT scanning at an interval of 3-months. We followed up all patients by telephone and based on outpatient records.

\section{Statistical analysis}

Statistical analysis was carried out by SPSS17.0 (Statistical Program for Social Sciences) software. Data were expressed as mean \pm standard deviation, and group comparison was performed by one-way analysis of variance or Wilcoxon test. Count data were expressed as value or percentage, and comparison between groups was performed by chi-square test or Fisher's exact test. $P$-values $<0.05$ were considered statistically significant.

\section{Results}

\section{Patient demographics}

There were total 95 (20.0\%) SGGNs in 474 solitary nodules from January 2008 to March 2015. Among the patients with SGGNs, 81 had adenocarcinoma and 14 had benign nodules. The clinicopathologic characteristics of 81 (17.1\%) patients with adenocarcinoma are summarized in Table 1. Among the 81 patients, $55(67.9 \%)$ were females and $26(32.1 \%)$ males, and the median age was 55.9 years (range $30-84$ years). Seventeen $(21 \%)$ patients were smokers and the rest nonsmokers. Four patients had a prior history of other tumors and eleven patients had complications such as COPD or asthma. Seventeen patients had a family history of cancer (such as liver cancer, gastric cancer, and colorectal cancer) and eight (9.9\%) patients had a family history of lung cancer. The number of patients whose blood tumor markers such as CEA, CA19-1, and NSE have elevated were 15 (18.3\%), 14 (17.1\%), and 17 $(20.7 \%)$ respectively. However, $15(3.0 \%)$ benign nodules were also reported, and the data are shown in Table S1.

Among the 81 patients with SGGNs, 12 (14.8\%) had AIS (Figure 1A and B), 14 (17.3\%) MIA (Figure 2A and B), and $55(67.9 \%)$ IA (Figure 3A and B). The predominant histologic 
Table I Characteristics of patients with GGNs

\begin{tabular}{|c|c|}
\hline Characteristics & $\begin{array}{l}\text { Number of } \\
\text { patients (\%) }\end{array}$ \\
\hline \multicolumn{2}{|l|}{ Sex } \\
\hline Female & $55(67.9)$ \\
\hline Male & $26(32.1)$ \\
\hline \multicolumn{2}{|l|}{ Age (45 years, I2) } \\
\hline Range (median) & $30-84(55.9)$ \\
\hline$<65$ & $53(65.4)$ \\
\hline$\geq 65$ & $28(34.6)$ \\
\hline \multicolumn{2}{|l|}{ Smoking habits } \\
\hline Current/former smoker & I7 (2I.0) (all males) \\
\hline Nonsmoker & $64(79.0)$ \\
\hline \multicolumn{2}{|l|}{ History of cancer } \\
\hline Yes & $4(4.9)$ \\
\hline No & $77(95.1)$ \\
\hline \multicolumn{2}{|l|}{ Family history of cancer } \\
\hline Yes/lung cancer & $17(21) / 8(9.9)$ \\
\hline No & $64(79)$ \\
\hline \multicolumn{2}{|l|}{ Complications } \\
\hline Yes & II (I3.6) \\
\hline No & $70(86.4)$ \\
\hline \multicolumn{2}{|l|}{ Blood tumor markers } \\
\hline CEA $(+)$ & $15(18.3)$ \\
\hline CAI $25(+)$ & $7(8.5)$ \\
\hline CAI9-9 (+) & $14(17.1)$ \\
\hline NSE $(+)$ & $17(20.7)$ \\
\hline CRFR2I-I (+) & $9(11.0)$ \\
\hline \multicolumn{2}{|l|}{ Surgical procedure } \\
\hline Lobectomy & $46(56.8)$ \\
\hline Sublobar resection & $21(25.9)$ \\
\hline Wedge resection & $14(17.3)$ \\
\hline \multicolumn{2}{|l|}{ Histologic types } \\
\hline $\mathrm{AAH}$ & $0(0.0)$ \\
\hline AIS & $12(14.8)$ \\
\hline MIA & $14(17.3)$ \\
\hline IPA & $55(67.9)$ \\
\hline \multicolumn{2}{|l|}{ Differentiation } \\
\hline Well-moderate & $80(98.7)$ \\
\hline Poor & $\mathrm{I}(\mathrm{I} .3)$ \\
\hline \multicolumn{2}{|l|}{ Pathologic T stage } \\
\hline IA & $30(37.0)$ \\
\hline IB & $51(63.0)$ \\
\hline \multicolumn{2}{|l|}{ Immunohistochemistry } \\
\hline ALK-V (+) & $2(2.5)$ \\
\hline ROSI (+) & $7(8.6)$ \\
\hline \multicolumn{2}{|l|}{ Nodular density } \\
\hline pGGNs & $35(43.2)$ \\
\hline mGGNs & $46(56.8)$ \\
\hline \multicolumn{2}{|l|}{ Locations (HRCT) } \\
\hline RUL & $35(43.2)$ \\
\hline RML & $4(4.9)$ \\
\hline RLL & $10(12.4)$ \\
\hline LUL & $26(32.1)$ \\
\hline LLL & $6(7.4)$ \\
\hline \multicolumn{2}{|l|}{ PET/CT (SUV value) } \\
\hline Normal & $4(40.0)$ \\
\hline$<2.5$ & $4(40.0)$ \\
\hline$\geq 2.5$ & $2(20.0)$ \\
\hline
\end{tabular}

Table I (Continued)

\begin{tabular}{ll}
\hline Characteristics & $\begin{array}{l}\text { Number of } \\
\text { patients (\%) }\end{array}$ \\
\hline Follow-up period, range (median), mo & $0.5-26(3.06)$ \\
Local recurrence or metastasis & 0 \\
\hline
\end{tabular}

Abbreviations: AIS, adenocarcinoma in situ; MIA, minimally invasive adenocarcinoma; IA, invasive pulmonary adenocarcinoma; pGGN, pure ground-glass opacity nodule; mGGN, mixed ground-glass opacity nodule; RUL, superior lobe of right lung; RML, middle lobe of right lung; RLL, inferior lobe of right lung; LUL, superior lobe of left lung; LLL, inferior lobe of left lung; ALK-V, anaplastic lymphoma kinase; PET, positron emission tomography, CT, computed tomography, HRCT, high-resolution computed tomography; mo, month; GGNs, ground-glass opacity nodules.

subtypes among 55 patients with IA were lepidic and acinar patterns $(n=29,52.7 \%)$ and five $(9.1 \%)$ patients had papillary patterns. All patients had stage I lung cancer. However, the rate of expression of anaplastic lymphoma kinase (ALK-V) and ROS-1 performed by immunohistochemistry was positive in $2.5 \%(\mathrm{n}=2)$ and $8.6 \%(\mathrm{n}=7)$ patients, respectively. The specificity and accuracy of HRCT of invasive lung adenocarcinoma were $85.2 \%$ and $87.4 \%$.

Furthermore, HRCT was performed and the results showed that there were 35 (43.2\%) pSGGNs and 46 (56.8\%) mSGGNs. SGGNs were primarily found to be located in the superior lobe of right lung $(n=35,43.2 \%)$ and then in the superior lobe of left lung $(n=26,32.1 \%)$. Preoperative positron emission tomography (PET)/CT scanning was carried out in 10 of the 81 patients $(12.3 \%)$, and only two patients' standard uptake value (SUV) was above 2.5 . The median follow-up period was 3.06 months. No recurrence or metastasis has been found until now.

\section{Comparison of imaging features}

Imaging features of the 81 SGGNs of different pathologic types are shown in Table 2 . The size was larger and the shape was much more irregular along with an increased malignant degree of lesions (from AIS to IA) ( $P=0.016$ and $P=0.027$, respectively). The density of IA nodules were more showed as mGGNs, however, AIS and MIA were more often showed as pGGNs on the contrary $(P<0.001)$. Pleural tag was observed more frequently in IA than in AIS and MIA $(P=0.018)$, but no difference was observed between AIS and MIA.

Furthermore, we compared the imaging features of pGGNs and mGGNs (Table 3). The results showed that mGGNs was significantly larger in size compared to pGGNs $(P<0.001)$. Besides the presence of spiculated, lobular, pleural tag, vascular cluster, bubble lucency, and air bronchogram were observed more frequently in mGGNs than in pGGNs (all $P<0.05$ ), the shape of mGGNs were more irregular $(P<0.001)$. 

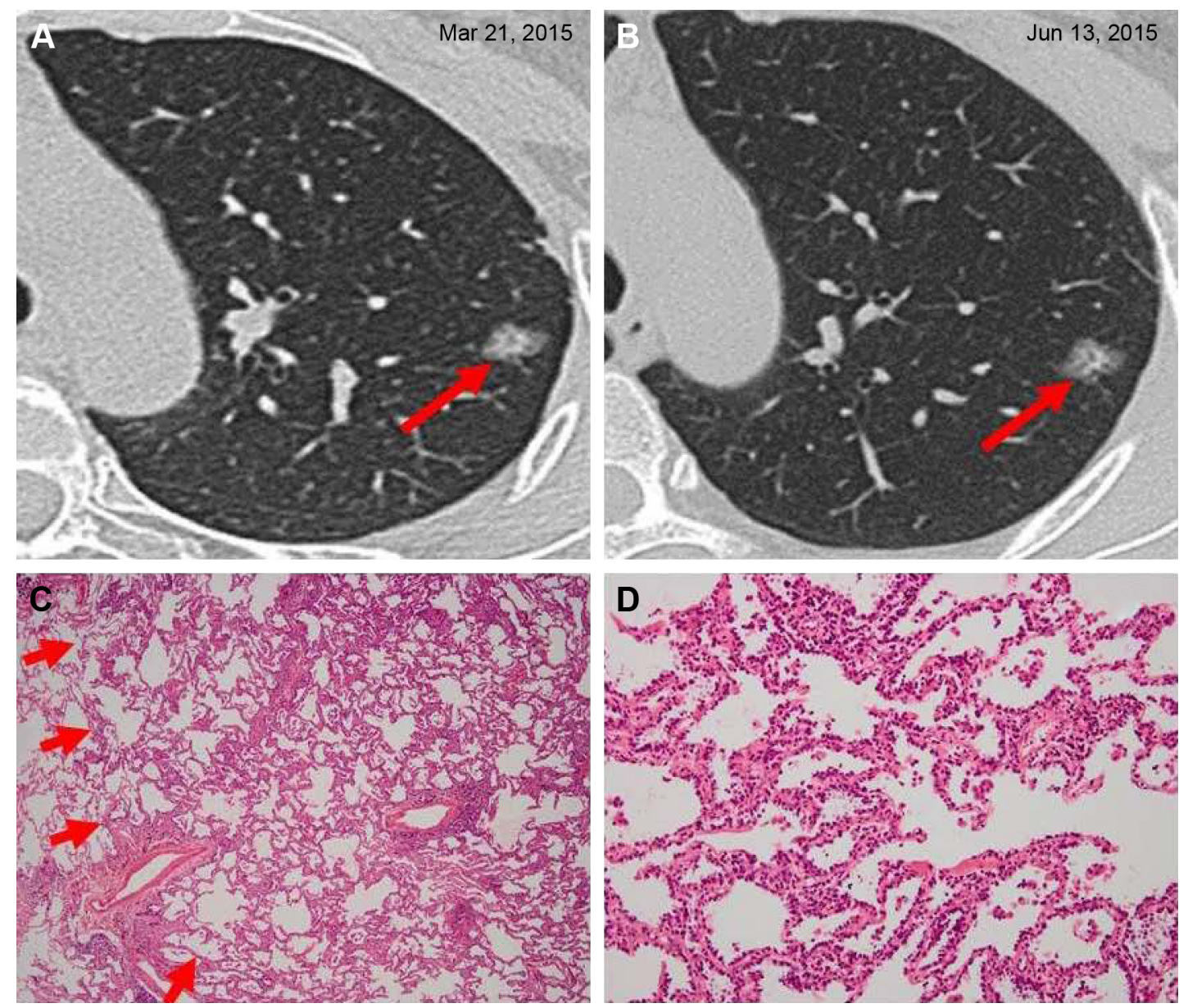

Figure I Adenocarcinoma in situ in a 44-year-old woman.

Notes: (A) A solitary ground-glass opacity nodule on the superior lobe of left lung was found at the time of her health checkup. She was a nonsmoker, did not have any individual history of cancer and no family history of cancer, and her blood tumor markers were negative. (B) On reexamination by HRCT after 3 months, no obvious change was noted. Hence, she decided to undergo surgical resection. Pathological diagnosis indicated that she had adenocarcinoma in situ. Immunohistochemistry: ALK-V (-), ROS-I (-). (C) Low-magnification (hematoxylin and eosin, original magnification $\times 40$ ) photomicrograph demonstrates uniform cuboidal cell proliferation (arrows) involving thickened alveolar walls (lepidic tumor growth). (D) High magnification of (C) (original magnification $\times 200$ ).

Abbreviations: ALK, anaplastic lymphoma kinase; HRCT, high-resolution computed tomography.

\section{Relationship between immunohistochemistry and pathologic subtypes}

Comparison of immunohistochemistry and pathologic subtypes showed that the expression of ALK-V and ROS-1 in different pathologic classifications had no statistical significance (Table 4).

In summary, the main results of this research are as follows: 1) among all the patients, with an average age of 55.9 years, the number of women who had lung adenocarcinoma with SGGNs images on HRCT was more than men, and most of them were nonsmokers and had no history of other cancers; 2) for the purpose of diagnosis and treatment, most of the patients underwent lobectomy, and blood tumor biomarkers such as CEA, CA19-9, and NSE were often found to be higher than normal; 3) most of the nodules were IA, and all the patients were at stage I and well differentiated; 4) none of the patients with SGGNs has tumor recurrence or metastasis until now; 5) the rate of positive expression of ALK-V and ROS-1 was very low, with $2.5 \%$ and $8.6 \%$, respectively; and 6 ) the specificity and accuracy of HRCT of invasive lung adenocarcinoma were $85.2 \%$ and $87.4 \%$. Based on the findings of imaging the following were concluded: 1) SGGNs was often found to be located in the superior lobe of right lung and then in the superior lobe of left lung; 2) results of PET/CT were often negative; and 3) nodule size was significantly larger in IA than in AIS and MIA, and the presence of pleural tag favored the diagnosis of IA.

\section{Discussion}

Our study explored the relationship between clinical pathological features and radiological characteristics of SGGNs on HRCT. Our results (malignant composition ratio was 

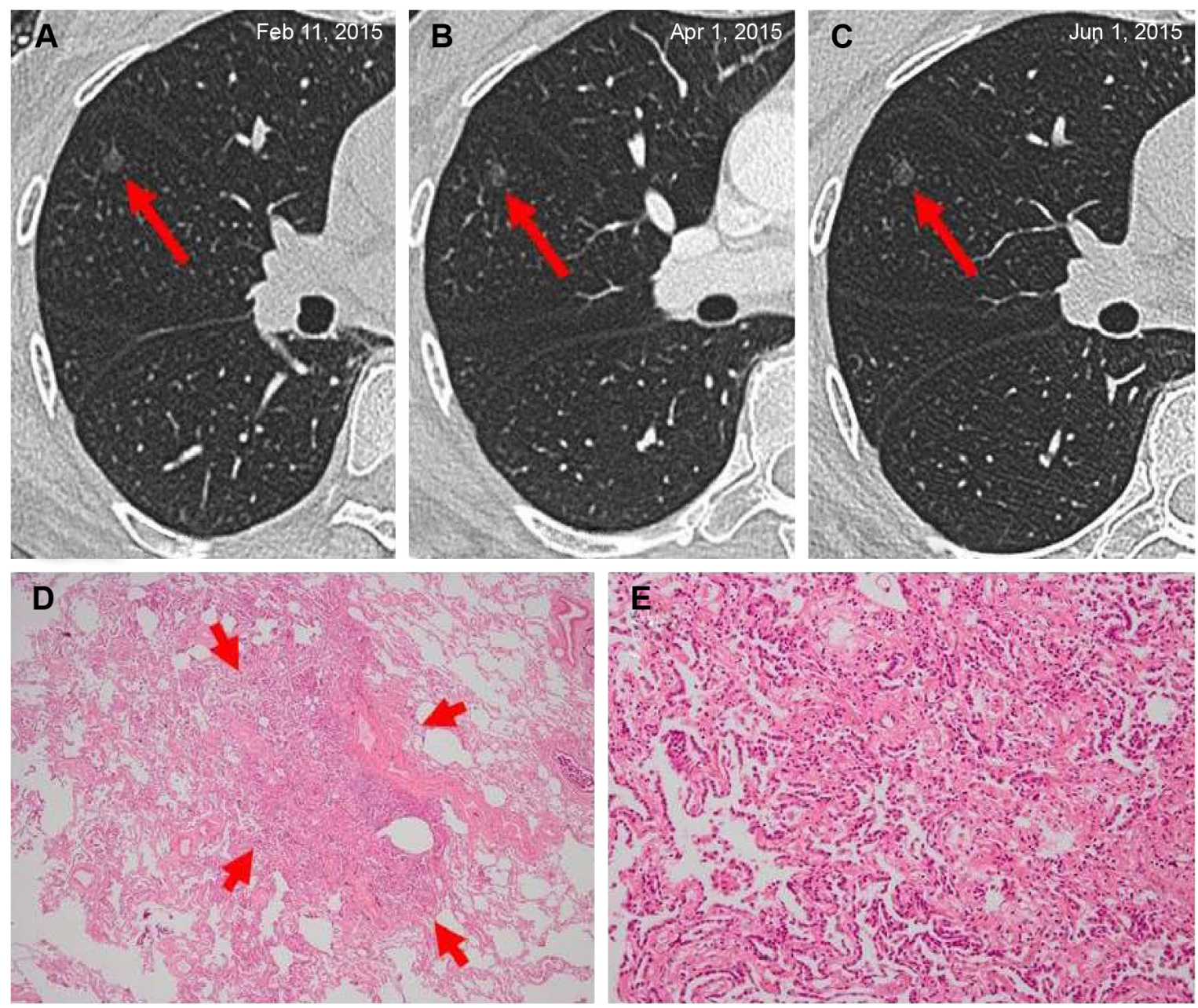

Figure 2 Minimally invasive adenocarcinoma in a 55-year-old woman.

Notes: (A) A solitary ground-glass opacity nodule on the superior lobe of right lung was observed at the time of her health checkup. She was a nonsmoker, did not have any individual history of cancer and no family history of cancer, and the level of her blood tumor markers CEA was $5.34 \mathrm{ng} / \mathrm{mL}$. She underwent reexamination by HRCT two times and no obvious change was noted (B and $\mathbf{C}$ ). Afterward, she decided to undergo surgical resection. Pathological diagnosis indicated that she had a minimally invasive adenocarcinoma. Immunohistochemistry: ALK-V (-), ROS-I (-). (D) Low magnification photomicrographs (hematoxylin and eosin, original magnification $\times 40$ ) demonstrates lesion consisting of predominantly lepidic tumor growth with several foci (arrows) of invasive acinar components $<5 \mathrm{~mm}$ in thickness. (E) High magnification of (D) (original magnification $\times 200$ ).

Abbreviations: ALK, anaplastic lymphoma kinase; HRCT, high-resolution computed tomography.

82.7\% [81/98] with 12 [14.8\%] AIS, 14 [17.3\%] MIA, and $55[67.9 \%]$ IA) are in accordance with a previous study, ${ }^{8}$ which showed that of the 330 surgically removed GGNs, malignant composition ratio was $95.2 \%$ (314/330), including 38 (12.1\%) AIS, 63 (20.1\%) MIA, and 213 (67.8\%) IA. But this is in contrast to the results of Lim et al, ${ }^{5}$ who showed that the main histologic type of GGNs in their research was AIS. Moreover, Lim et $\mathrm{al}^{5}$ showed that the size (cutoff $=16.4 \mathrm{~mm}, P=0.032$ ), mass (cutoff $=0.472 \mathrm{~g}$, $P=0.040)$, and bronchogram $(P=0.012)$ of a nodule are determinants of IA in persistent $\mathrm{pGGNs}$ with a diameter $\geq 10 \mathrm{~mm}$. However, our study showed that the size, shape, density, and the presence of pleural tag were statistically significant for SGGNs compared to other histological types. In addition, Lee et $\mathrm{al}^{9}$ studied $80 \mathrm{GGNs}$ and found that lobular marginal appearance of the nodules was an independent risk factor for malignancy ( $\mathrm{OR}=13.769, P=0.016$ ). But another study by Kim et $\mathrm{al}^{10}$ carried out on 53 GGNs suggested that there was no relationship between marginal features of GGNs and degree of malignancy. Furthermore, some studies indicated that nodules with a maximum diameter of $>8 \mathrm{~mm}$ and presence of solid element in the nodules, respectively, were independent risk factors for malignancy ${ }^{11}$ and lymph node metastasis (OR $=4.87,95 \%$ confidence interval 1.51-15.77). ${ }^{12}$

PET/CT scanning did not show any differentiation between malignancy and benign GGNs. In 53 patients with GGNs, Tsushima et al ${ }^{13}$ found that the average and maximum SUV values obtained for benign lesions were significantly higher than that obtained for malignant lesions. Sensitivity, 

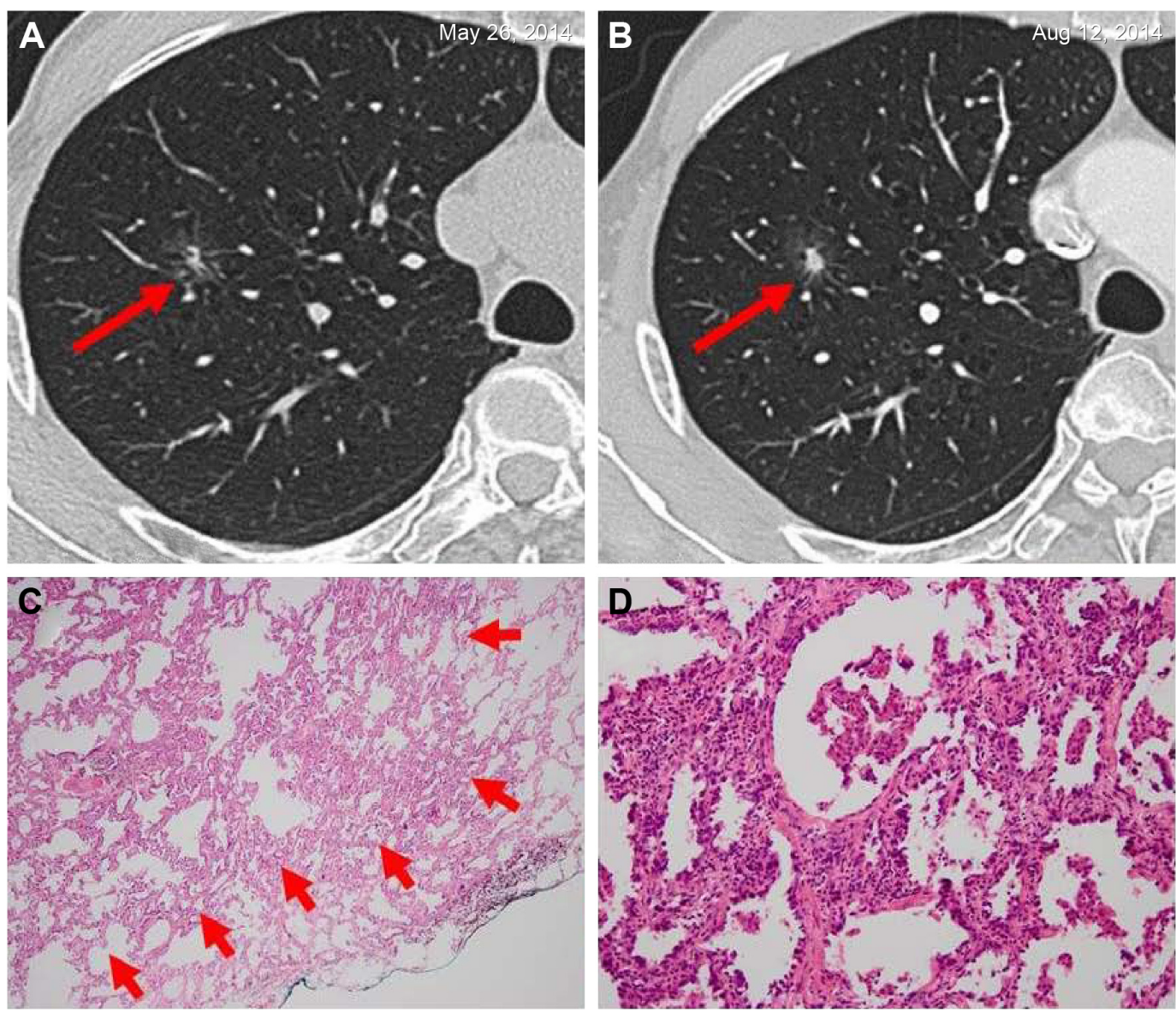

Figure 3 Invasive adenocarcinoma in a 66-year-old man.

Notes: (A) A solitary ground-glass opacity nodule on the superior lobe of right lung was observed at the time of his medical examination. He had a smoking history of more than 30 years and no individual history of other cancers; his father had colorectal cancer, and his blood tumor markers were negative. The solid composition of the nodule was found to be increased when he was reexamined by HRCT after 3 months (B). Therefore, he decided to undergo surgical resection. Pathological diagnosis indicated that it was an invasive adenocarcinoma. Immunohistochemistry: ALK-V (-), ROS-I (-). (C) Low magnification (hematoxylin and eosin, original magnification $\times 40)$ shows round-to oval-shaped invasive adenocarcinoma (arrows). (D) High magnification of (C) (original magnification $\times 200)$.

Abbreviations: ALK, anaplastic lymphoma kinase; HRCT, high-resolution computed tomography.

Table 2 Imaging features of GGNs classified into different histologic types

\begin{tabular}{|c|c|c|c|c|}
\hline Characteristics & AIS $(n=12)$ & $\operatorname{MIA}(n=14)$ & IA $(n=55)$ & $P$-value \\
\hline Size (mean \pm standard deviation, $\mathrm{cm}$ ) & $1.45 \pm 0.60$ & $1.49 \pm 0.55$ & $2.00 \pm 0.87$ & $0.016^{*}$ \\
\hline Density (n) & & & & $<0.00 I^{*}$ \\
\hline pGGNs & 10 & 9 & 16 & \\
\hline mGGNs & 2 & 5 & 39 & \\
\hline Shape (n) & & & & $0.027^{* *}$ \\
\hline Round & 8 & 6 & 15 & \\
\hline Oval & I & 2 & 4 & \\
\hline Irregular & 3 & 6 & 36 & \\
\hline Margin (n) & & & & 0.190 \\
\hline Smooth & 10 & 10 & 28 & \\
\hline Lobular & 1 & 2 & 8 & \\
\hline Spiculated & I & 0 & 14 & \\
\hline Lobular and spiculated & 0 & 2 & 5 & \\
\hline Pleural tag (n) & 1 & 2 & 26 & $0.007^{*}$ \\
\hline Vascular cluster (n) & 0 & 2 & 13 & 0.146 \\
\hline Bubble-like sign (n) & 3 & 5 & 23 & 0.542 \\
\hline Air bronchogram $(n)$ & I & 3 & 13 & 0.498 \\
\hline
\end{tabular}

Notes: *IA vs AIS and MIA statistically significant; **IA vs AIS statistically significant.

Abbreviations: GGN, ground-glass opacity nodule; AIS, adenocarcinoma in situ; MIA, minimally invasive adenocarcinoma; IA, invasive pulmonary adenocarcinoma; pGGN, pure ground-glass opacity nodule; mGGN, mixed ground-glass opacity nodule. 
Table 3 Imaging features of GGNs classified into pGGNs and mGGNs

\begin{tabular}{|c|c|c|c|}
\hline Characteristics & pGGNs & mGGNs & $P$-value \\
\hline $\begin{array}{l}\text { Size (mean } \pm \text { standard } \\
\text { deviation, } \mathrm{cm} \text { ) }\end{array}$ & $\mid .37 \pm 0.5 \mathrm{I}$ & $2.17 \pm 0.83$ & $<0.00 I^{*}$ \\
\hline Locations (HRCT) & & & 0.056 \\
\hline RUL & 10 & 25 & \\
\hline RML & 1 & 3 & \\
\hline RLL & 5 & 5 & \\
\hline LUL & 17 & 9 & \\
\hline LLL & 2 & 4 & \\
\hline Shape (n) & & & $<0.00 I^{*}$ \\
\hline Round & 26 & 3 & \\
\hline Oval & 4 & 3 & \\
\hline Irregular & 5 & 40 & \\
\hline Margin (n) & & & $0.001 *$ \\
\hline Smooth & 30 & 18 & \\
\hline Lobular & 2 & 9 & \\
\hline Spiculated & 2 & 12 & \\
\hline Lobular and spiculated & 1 & 7 & \\
\hline Pleural tag (n) & 4 & 25 & $<0.00 I^{*}$ \\
\hline Vascular cluster (n) & 3 & 12 & $0.015^{*}$ \\
\hline Bubble lucency (n) & 6 & 25 & $<0.00 I^{*}$ \\
\hline Air bronchogram (n) & 0 & 17 & $<0.00 I^{*}$ \\
\hline
\end{tabular}

Note: *Statistically significant.

Abbreviations: pGGN, pure ground-glass opacity nodule; mGGN, mixed groundglass opacity nodule; HRCT, high-resolution computed tomography; RUL, superior lobe of right lung; RML, middle lobe of right lung; RLL, inferior lobe of right lung; LUL, superior lobe of left lung; LLL, inferior lobe of left lung.

specificity, and accuracy of the diagnosis of benign lesions were $100.0 \%, 96.4 \%$, and $100.0 \%$, respectively, when SUV value was $>1.5$. Our results are found to be consistent with prior studies. Besides, it may reduce the sensitivity of PET/CT in diagnosis of malignant lesions because of lymph node metastasis and distant metastasis is not occurred in GGNs.

However, Naidich et $\mathrm{a}^{14}$ in their study reported the following: 1) no CT follow-up is required for solitary pGGNs when the diameter of GGNs $\leq 5 \mathrm{~mm}$; 2) the patient has to be initially followed up for CT at 3 months to confirm persistence and then followed up annually for $\mathrm{CT}$ for a minimum of 3 years; PET/CT is of limited value; and 3 ) the patient has to be initially followed up for $\mathrm{CT}$ at 3 months to confirm persistence. If persistent and solid component $<5$ $\mathrm{mm}$ is observed, then the patient should be annually followed up for CT for a minimum of 3 years. If persistent and solid component $\geq 5 \mathrm{~mm}$ is found, then biopsy or surgical resection is required. PET/CT should be considered for part-solid nodules $>5 \mathrm{~mm}$. The results of our study are consistent with that of Naidich et al.

We also analyzed the relationship between the expression of ALK-V and ROS-1 among different histologic types. The results showed that ALK-V and ROS-1 were expressed in only IA, and positive expression rates were only $2.5 \%$ and $8.6 \%$, respectively.

Our study had several limitations. First, it was a retrospective research, and the number of patients was relatively small as we analyzed only patients with SGGNs. Second, the median follow-up period was too short, only 3.06 months, as most of the patients did not want a long follow-up period and decided on surgical resection after no more than two reexaminations or even without any reexamination. Third, we included nodules with a diameter $\leq 30 \mathrm{~mm}$, which may have contributed to a selection bias. Finally, variations in nodule measurement and characterization of lesions might have been possible due to different observers.

\section{Conclusion}

The size, density, shape, and pleural tag of a nodule are determinants ofIA in SGGNs with diameter $\leq 30 \mathrm{~mm}$ on HRCT, and detection of ALK-V expression and performance of PET/ CT scan are not preferred as routine examinations.

\section{Acknowledgments}

This work was supported by the National Natural Science Foundation of China (No 81372504), the Science and Technology Support Program of Science and Technology Department of Sichuan Province (2014SZ-0148), and the International Cooperation Program of Science and Technology Department of Sichuan Province (2014AA022202-2).

\section{Author contributions}

ZXQ and WML conceived and designed the experiments. ZXQ and YC performed the experiments. ZXQ, YC, WYW, $\mathrm{XW}$, and WLW analyzed the data. WYW, XW, and WLW

Table 4 Comparison of immunohistochemistry and pathologic subtypes

\begin{tabular}{|c|c|c|c|c|c|c|}
\hline \multirow[t]{2}{*}{ Pathologic subtypes } & \multicolumn{3}{|l|}{ ALK-V } & \multicolumn{3}{|l|}{ ROS-I } \\
\hline & Negative & Positive & $P$-value & Negative & Positive & $P$-value \\
\hline AIS & 12 & 0 & 0.616 & 12 & 0 & 0.216 \\
\hline MIA & 14 & 0 & & 14 & 0 & \\
\hline IA & 53 & 2 & & 49 & 6 & \\
\hline
\end{tabular}

Abbreviations: AIS, adenocarcinoma in situ; MIA, minimally invasive adenocarcinoma; IA, invasive pulmonary adenocarcinoma; ALK-V, anaplastic lymphoma kinase. 
contributed reagents/materials/analysis tools. ZXQ and DL wrote the paper. All authors contributed toward data analysis, drafting and critically revising the paper and agree to be accountable for all aspects of the work.

\section{Disclosure}

All authors declare no conflicts of interest in this work.

\section{References}

1. Austin JH, Muller NL, Friedman PJ, et al. Glossary of terms for CT of the lungs: recommendations of the Nomenclature Committee of the Fleischner Society. Radiology. 1996;200(2):327-331.

2. Collins J, Stern EJ. Ground-glass opacity at CT: the ABCs. AJR Am J Roentgenol. 1997;169(2):355-367.

3. Lee HY, Lee KS. Ground-glass opacity nodules: histopathology, imaging evaluation, and clinical implications. $J$ Thorac Imaging. 2011; 26(2):106-118.

4. Henschke CI, Yankelevitz DF, Mirtcheva R, et al. CT screening for lung cancer: frequency and significance of part-solid and nonsolid nodules. AJR Am J Roentgenol. 2002;178(5):1053-1057.

5. Lim HJ, Ahn S, Lee KS, et al. Persistent pure ground-glass opacity lung nodules $\geq 10 \mathrm{~mm}$ in diameter at CT scan: histopathologic comparisons and prognostic implications. Chest. 2013;144(4):1291-1299.

6. Chang B, Hwang JH, Choi YH, et al. Natural history of pure groundglass opacity lung nodules detected by low-dose CT scan. Chest. 2013;143(1):172-178.
7. Travis WD, Brambilla E, Noguchi M, et al. International association for the study of lung cancer/american thoracic society/european respiratory society international multidisciplinary classification of lung adenocarcinoma. J Thorac Oncol. 2011;6(2):244-285.

8. Cho J, Ko SJ, Kim SJ, et al. Surgical resection of nodular ground-glass opacities without percutaneous needle aspiration or biopsy. BMC Cancer. 2014;14:838.

9. Lee HJ, Goo JM, Lee CH, et al. Predictive CT findings of malignancy in ground-glass nodules on thin-section chest CT: the effects on radiologist performance. Eur Radiol. 2009;19(3):552-560.

10. Kim HY, Shim YM, Lee KS, Han J, Yi CA, Kim YK. Persistent pulmonary nodular ground-glass opacity at thin-section CT: histopathologic comparisons. Radiology. 2007;245(1):267-275.

11. Heo EY, Lee KW, Jheon S, Lee JH, Lee CT, Yoon HI. Surgical resection of highly suspicious pulmonary nodules without a tissue diagnosis. Jpn J Clin Oncol. 2011;41(8):1017-1022.

12. Matsuguma H, Yokoi K, Anraku M, et al. Proportion of ground-glass opacity on high-resolution computed tomography in clinical T1 N0 M0 adenocarcinoma of the lung: a predictor of lymph node metastasis. J Thorac Cardiovasc Surg. 2002;124(2):278-284.

13. Tsushima Y, Tateishi U, Uno H, et al. Diagnostic performance of $\mathrm{PET} / \mathrm{CT}$ in differentiation of malignant and benign non-solid solitary pulmonary nodules. Ann Nucl Med. 2008;22(7):571-577.

14. Naidich DP, Bankier AA, MacMahon H, et al. Recommendations for the management of subsolid pulmonary nodules detected at CT: a statement from the Fleischner Society. Radiology. 2013;266(1):304-317. 


\section{Supplementary material}

Table SI Comparison of features between patients with benign GGNs and adenocarcinomas

\begin{tabular}{|c|c|c|}
\hline \multirow[t]{2}{*}{ Characteristics } & \multicolumn{2}{|c|}{ Number of patients (\%) } \\
\hline & Benign GGNs (14) & Adenocarcinomas (8I) \\
\hline \multicolumn{3}{|l|}{ Sex } \\
\hline Female & $9(64.3)$ & $55(67.9)$ \\
\hline Male & $5(35.7)$ & $26(32.1)$ \\
\hline \multicolumn{3}{|l|}{ Age (45 years, I2) } \\
\hline Range (median) & 38-75 (57.I) & 30-84 (55.9) \\
\hline$<65$ & II (78.6) & $53(65.4)$ \\
\hline$\geq 65$ & $3(21.4)$ & $28(34.6)$ \\
\hline \multicolumn{3}{|l|}{ Smoking habits } \\
\hline Current/former smoker & 3 (2I.4) (all males) & I7 (2। .0) (all males) \\
\hline Nonsmoker & II (78.6) & $64(79.0)$ \\
\hline \multicolumn{3}{|l|}{ History of cancer } \\
\hline Yes & I (7.I) & $4(4.9)$ \\
\hline No & $13(92.9)$ & $77(95.1)$ \\
\hline \multicolumn{3}{|l|}{ Family history of cancer } \\
\hline Yes/lung cancer & 0 & $17(2 \mid) / 8(9.9)$ \\
\hline No & 14 & $64(79)$ \\
\hline \multicolumn{3}{|l|}{ Blood tumor markers } \\
\hline CEA $(+)$ & 0 & $15(18.3)$ \\
\hline CAI $25(+)$ & $\mathrm{I}(0.1)$ & $7(8.5)$ \\
\hline CAI9-9 (+) & 0 & $14(17.1)$ \\
\hline NSE $(+)$ & $\mathrm{I}(0.1)$ & $17(20.7)$ \\
\hline CRFR2I-I (+) & 0 & $9(11.0)$ \\
\hline \multicolumn{3}{|l|}{ Nodular density } \\
\hline pGGNs & $9(64.3)$ & $35(43.2)$ \\
\hline mGGNs & $5(35.7)$ & $46(56.8)$ \\
\hline Size (mean \pm standard deviation, $\mathrm{cm}$ ) & $0.88 \pm 0.46$ & $1.98 \pm 0.85$ \\
\hline \multicolumn{3}{|l|}{ Locations (HRCT) } \\
\hline RUL & $3(2 \mid .4)$ & $35(43.2)$ \\
\hline RML & $3(2 \mid .4)$ & $4(4.9)$ \\
\hline RLL & $2(14.3)$ & $10(12.4)$ \\
\hline LUL & $5(35.7)$ & $26(32.1)$ \\
\hline LLL & $\mathrm{I}(7.1)$ & $6(7.4)$ \\
\hline
\end{tabular}

Abbreviations: GGNs, ground-glass opacity nodule; pGGN, pure ground-glass opacity nodule; mGGN, mixed ground-glass opacity nodule; RUL, superior lobe of right lung; RML, middle lobe of right lung; RLL, inferior lobe of right lung; LUL, superior lobe of left lung; LLL, inferior lobe of left lung; NSE, neuron specific enolase.

\section{Publish your work in this journal}

Therapeutics and Clinical Risk Management is an international, peerreviewed journal of clinical therapeutics and risk management, focusing on concise rapid reporting of clinical studies in all therapeutic areas, outcomes, safety, and programs for the effective, safe, and sustained use of medicines. This journal is indexed on PubMed Central, CAS,
EMBase, Scopus and the Elsevier Bibliographic databases. The manuscript management system is completely online and includes a very quick and fair peer-review system, which is all easy to use. Visit http://www.dovepress.com/testimonials.php to read real quotes from published authors.

Submit your manuscript here: http://www.dovepress.com/therapeutics-and-clinical-risk-management-journal 